\title{
CEP 16-04
}

\section{Information Asymmetry and Risk Transfer Markets}

\author{
Eric Stephens \\ Carleton University \\ James R. Thompson \\ University of Waterloo
}

March 2016

\section{CARLETON ECONOMIC PAPERS}
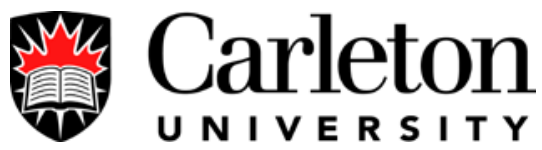

Department of Economics

1125 Colonel By Drive

Ottawa, Ontario, Canada

$$
\text { K1S 5B6 }
$$




\title{
Information Asymmetry and Risk Transfer Markets
}

\author{
Eric Stephens \\ Carleton University \\ eric.stephens@carleton.ca
}

\author{
James R. Thompson* \\ University of Waterloo \\ james@uwaterloo.ca
}

March 17, 2016

\begin{abstract}
We provide a tractable model of counterparty risk in an intermediated risk transfer market, and analyze the consequences of this risk being private information. We show that unknown type information can be revealed when large trades are observable; however, the allocation is shown to be constrained inefficient. The inefficiency is highlighted by considering the imposition of a transaction tax, which can improve welfare by encouraging more information revelation and increasing risk transfer. The results suggest that increased transparency and/or central counterparty arrangements in over-the-counter derivative markets may promote transparency of counterparty risk.
\end{abstract}

Keywords: Risk Transfer Markets, Asymmetric Information, Counterparty Risk, Regulation

\footnotetext{
*We would like to thank Viral Acharya, Bruno Biais, Robin Boadway, Neil Brisley, Jamie McAndrews, Duane Seppi, Hans Stoll, Javier Suarez, as well as seminar participants at the 2015 FIRS meetings (Iceland), 2014 Western Finance Association meetings (Monterey), Federal Reserve Board of Governors, the Wharton School, Vanderbilt University, Georgia State University (RMI), McMaster University, Queen's University, Reserve Bank of New Zealand, Bank of Canada, and the Center for Economic Studies (Munich). Financial support provided by SSHRC Grant 4352013-0180 is gratefully acknowledged. Addresses: C-870 Loeb Building, 1125 Colonel By Drive, Ottawa, Ontario, Canada, K1S 5B6, phone: +1 613-520-2600 x 8132 (E. Stephens). 200 University Avenue West, Waterloo, Ontario, Canada N2L 3G1, phone: +1 519-888-4567 x 38601 (J. Thompson)
} 


\section{Introduction}

Over the past thirty years the market for risk transfer has grown considerably. For example, over-the-counter (OTC) derivative contracts grew from a notional size of 100 trillion dollars in 2001 to 614 trillion dollars as of $2007 .{ }^{1}$ Further, potential inefficiencies in these markets have been front and center in the discussion over market reform following the recent financial crisis. A key difference between risk transfer markets and traditional asset markets is the potential for counterparty risk. In a standard asset market transaction, a buyer purchases an asset from a seller and the value of the asset is not dependent on the risk of the seller. On the other hand, risk transfer contracts specify contingent payments, the value of which depends on the counterparty being solvent when a payment is required. For example, in a credit default swap (CDS) contract, the buyer purchases protection against the event that some underlying debt will default. If the party which sold that protection becomes insolvent, the contract loses value, independent of the quality of the underlying debt. Furthermore, counterparty risk can be a source of asymmetric information, since the counterparty is likely to know more about its own risk than the party with which it is contracting. This presents a new avenue of study and is our key departure from the extant literature on financial markets.

We model an intermediated/dealer risk transfer market with asymmetric information over counterparty quality. With simple contingent contracts written on an underlying risk, we allow for one party who can default on their obligation and consider an environment in which counterparty quality may be unknown. Given the opacity of OTC markets (e.g., see Acharya and Bisin (2014)), we assume that position size is generally not observed and thus non-contractible; however, it is known whether positions exceed some large threshold. This assumption captures an important feature of risk transfer markets, which can arise for a number of reasons. For example, under Dodd-Frank (and the EMIR in Europe), a growing number of OTC markets now mandate large trader revelation, wherein parties with a contract size over some threshold are required to report this to the regulator, who in turn makes that information available to market participants. ${ }^{2}$ Similar arrangements are being considered in many more OTC markets such as those for CDS. ${ }^{3}$ Alternatively, in

\footnotetext{
${ }^{1}$ Deutsche Börse Group, "The Global Derivatives Market: An Introduction", White Paper (2008), BIS "Triennial Central Bank Survey: Foreign Exchange and Derivative Market Activity in 2001", Basel: Bank for International Settlements (2002)).

${ }^{2}$ For example, see http://www.cftc.gov/LawRegulation/FederalRegister/FinalRules/2011-18054

${ }^{3}$ See for example Chen et al. (2011).
} 
the absence of mandatory large trader reporting, we can interpret this assumption as the ability of players to hide exposures in their financial statements, but only for relatively small positions. This assumption can also be viewed as a reduced form way of capturing the possibility that buyers may infer something about sellers' total positions through their own contracts when there are relatively few dealers, similar to the analysis in Leitner $(2012) .{ }^{4}$ Consider as an example the OTC dealer CDS market, which is dominated by 4 dealers who comprise $50 \%$ of activity, with $95 \%-98 \%$ of trades having one of 14 dealers (refereed to as the G14 dealers) involved (Chen et al. (2011)). Potential sellers are free to contract with multiple dealers if they wish. For smaller contract sizes, it is unlikely that any dealer would learn the aggregate number of contracts that a seller has sold. When desired positions are large however, it is likely that information can be gained by dealers who can share/exchange some information outright or by an individual dealer inferring the aggregate contract size given that there are a limited number of dealers with which to split contracts over.

Since position size is not generally contractible, a separating equilibrium as described in Rothschild and Stiglitz (1976) cannot be achieved. However, in contrast to classic market-breakdown results like that first presented in Akerlof (1970), we show that when large traders can be identified, some risky counterparties may freely reveal themselves, despite facing worse prices when they do so. Potential information revelation notwithstanding, we show that the competitive equilibrium is (constrained) inefficient, since some risky counterparties still choose to keep their type information hidden. We show that a transaction tax on pooling counterparties can increase efficiency by encouraging more revelation. When positions are opaque, so that there is no information on how many contracts counterparties have written, only pooling can be supported in equilibrium. Thus, proposals designed to increase position transparency, including central counterparty arrangements, can actually increase transparency of counterparty risk as well.

To capture the intuition behind our results, consider two types of risk averse agents: those with positive (or upside) exposure to some risk, and those with negative (or downside) exposure. In other words, one type of agent benefits if some risky event occurs, while the other type suffers a loss. We refer to parties with positive exposure as sellers and those with negative exposure as buyers. Assume that sellers come in two types: risky and safe, differentiated by their counterparty risk.

\footnotetext{
${ }^{4}$ Leitner (2012) shows that under some conditions, a situation in which market participants learn whether an agent's position is above some threshold or below some threshold arises endogenously. In contrast to our model, in his setting there is private information about an agent's position but not underlying type.
} 
Parties use a contingent contract wherein all payments occur ex-post and all contracts are made with a risk neutral competitive market maker. With full information, risky sellers receive a lower price than safe sellers since counterparty risk is priced. With asymmetric information, risky sellers have a choice of whether to pool with safe sellers, or to reveal themselves. For any given price, risky counterparties will desire larger contract sizes than their safe counterparts due to limited liability. Therefore, if risky sellers pool with safe sellers, they are forced to supply less than they would like to at the pooling price in order to stay hidden. This tension creates an incentive for some risky sellers to deviate and reveal themselves; by doing so they will be able to trade more, albeit at a lower price. Even though the competitive equilibrium may feature some risky sellers fully revealing, the allocation is shown to be information constrained inefficient, since risky sellers do not internalize the cost that pooling has on safe sellers. In addition, pooling risky sellers do not internalize the effect that the contraction in supply due to pooling has on buyers. In particular, when the market maker has limits on the amount of inventory it can hold, the contraction in supply leads to an inefficiently low amount of risk transfer for buyers. ${ }^{5}$ A social planner can effectively cross subsidize revealing and pooling risky sellers because it is not restricted by the competition environment of the market maker. Doing so can generate more revealing risky sellers thereby increasing welfare. A Pareto improving policy is to introduce a transaction tax on the pool of unknown sellers, thereby encouraging more revelation.

In a completely opaque market, only a pooling equilibrium can prevail since risky sellers can obtain large contract sizes without being detected. Our results therefore have suggestive implications for policies that can provide position transparency such as trade reporting and central counterparties (CCPs). In particular, CCP's and/or regulators could potentially use position size to endogenously promote information revelation, thereby improving information about the risk of its members.

\section{Related Literature}

We contribute to the literature on incentives and counterparty risk and the financial markets

\footnotetext{
${ }^{5}$ Shachar (2012) provides empirical evidence that dealers do become averse to inventory risk in the presence of counterparty risk.
} 
literature. Thompson (2010), Acharya and Bisin (2014), and Biais, Heider and Hoerova (2014) demonstrate moral hazard problems that may be present on the sell side of the market wherein the seller may take positions which increase counterparty risk and are not in the best interest of the buyer. In contrast, we analyze an asymmetric information problem on counterparty quality. Stephens and Thompson (2014a) analyze exogenously given differences in counterparty risk as in this paper, but do not consider differences in the type of risk being transferred as here. Importantly, the driving force behind the results of our paper come from a contract size decision, whereas the contract size is exogenous in Stephens and Thompson (2014a). Incorporating a contract size choice allows us to create a market and study the welfare implications of asymmetric information, both of which are not possible in Stephens and Thompson (2014a).

The financial markets literature has explored the asymmetric information problem of assets in great detail. In contrast, we study the asymmetric information problem of counterparty risk while we assume that the underlying risk (which is analogous to the asset risk studied in previous literature) is known. Most relevant to our analysis are papers that model trade size and asymmetric information. For example, Easley and O'Hara (1987) show that parties with insider information may be willing to trade large quantities, however only a partial separating equilibrium can be supported wherein some uninformed parties must also be willing to trade large sizes. Seppi (1990) extends this argument and shows why uninformed traders may endogenously wish to trade large quantities, even if this leads to less favorable price quotes as the market maker believes they are more likely to be informed. One can draw a parallel between our risky and safe sellers and the informed and uninformed agents from Easley and O'Hara (1987). In that paper, if there were no uninformed traders who trade large quantities, the informed trader would be revealed and lose all profits from superior information. In contrast, we can achieve full separation in that some risky sellers trade large quantities despite no safe sellers wishing to do so. In addition, our general risk transfer environment (as opposed to the insider trading setting of those papers) allows for the introduction of counterparty risk, a welfare analysis, and new policy implications.

Non-exclusivity of contracts plays an important role in our paper, which contributes to the literature in this area (see for example, Attar et al. (2011), Ales and Maziero (2014), and Stephens and Thompson (2014b)). Exclusivity provides contractible information to a principal about an agent's allocation. Even in the absence of exclusivity, if the principal has full information about 
the allocations of agents, this can be equivalent to the case of exclusive contracts. In our case, there is some contractible information about the agent's position, i.e., large positions are observable. Non-exclusive contracts are typically implemented with linear prices (i.e., there can be no menus because only price is observed), which is true in our environment everywhere except at the point at which large positions are revealed. Thus, in a sense our environment is neither entirely exclusive nor non-exclusive. This represents a key difference between our paper and the literature on contract exclusivity. For example, Attar et al. (2011) and Ales and Maziero (2014) show that the equilibrium can feature a market breakdown similar to that in Akerloff (1970), wherein only risky types participate. In contrast, the ability to observe large traders allows for an equilibrium in which both risky and safe types participate, and ex-ante identical risky types may even trade different amounts (for different prices).

The rest of the paper is organized as follows. Section 2 outlines the model, while Section 3 characterizes the competitive equilibrium and the social planner's problem with full information. Section 4 analyzes the competitive equilibrium and the social planner's problem with asymmetric information on seller type. Section 5 considers restrictions to inventory and Section 6 concludes. Section 7 contains a discussion on robustness and the Appendix contains non-trivial proofs.

\section{The Model}

We construct a model of buyers, sellers, and market markers. In Section 3, we analyze the case of full information, so all of the variables to be introduced below are assumed to be common knowledge. We revisit this in Section 4.

\subsection{Market Participants}

Excluding the market maker, all agents are endowed with wealth $w$ and have preferences characterized by a strictly increasing, strictly concave utility function $u(x)$. There are two forms of heterogeneity among agents. First, with some probability an event denoted $D$ ('Default') occurs which results in measure $N_{B}$ agents suffering a loss $L>0$, while $N_{S}$ agents gain $L$. We denote the state in which the event does not occur $N D$ ('No Default'). To fix ideas, this risk can be thought of as an asset (or assets) that defaults and that some parties have positive exposure (will experience 
a gain if the event occurs) while others have negative exposure (will experience a loss if the event occurs). This creates a natural environment to study risk transfer since there will be gains to trade for both parties from contracting. We refer to those facing a potential loss as (B)uyers and gain as (S)ellers.

The second source of heterogeneity among agents is counterparty risk, which we capture by allowing for sellers that are either (r)isky or (s)afe. ${ }^{6}$ Safe sellers always meet their obligations, while risky sellers are only solvent in some states. Specifically, risky sellers may receive a shock which wipes out their endowment and gain $L$ such that they are unable to meet their obligations and receive a payoff of zero. Denote the no shock event as $N F$ ('No Failure') and the shock event as $F$ ('Failure'). Thus, we use the word 'Default' to refer to the underlying event and 'Failure' to refer to the counterparty. To achieve our results with the minimum heterogeneity between seller types, we assume that the joint probability of $N D$ and $F$ is zero. ${ }^{7}$ We define joint probabilities $\pi_{i}>0$, where $i \in\{1,2,3\}$ represents the three possible states of the world as follows: $\pi_{1}=\operatorname{prob}(N D)$, $\pi_{2}=\operatorname{prob}(D, N F)$ and $\pi_{3}=\operatorname{prob}(D, F)$. Thus, $\pi_{1}$ is the probability that no loss/gain occurs for the buyer/sellers, $\pi_{2}$ the probability that a loss (gain) occurs for buyers (sellers) and no sellers fail, and $\pi_{3}$ the probability that a loss (gain) occurs for buyers (safe sellers) and risky sellers fail. Finally, let $N_{S r}$ and $N_{S s}$ denote the number of risky and safe sellers respectively, so that $N_{S}=N_{S r}+N_{S s}$.

\subsection{Market Maker}

To model an intermediated market, we assume the existence of a competitive measure one of market makers who serve as the buyer to the sellers and seller to the buyers. To pin down the price, we will assume Bertrand competition among market makers. We assume that a market maker is not restricted on the amount of inventory that can be carried. This assumption is relaxed in Section 5. For ease of exposition, we will consider buyers and sellers interacting with one market

\footnotetext{
${ }^{6}$ We assume that only our sellers are subject to the risk of failure to simplify the analysis. One could imagine that the buyer posts the asset as collateral. Alternatively, although we will introduce our risk transfer as a contingent contract, our results will obtain if there was an upfront payment by buyers in exchange for a contingent payment from the seller, such as in a credit default swap contract. In this case, the payment from the buyer to the seller would be assured.

${ }^{7}$ Without this assumption, in the risk transfer contract that we will introduce below, risky sellers will wish to contract not only to smooth consumption across the gain and no gain states, but also to smooth consumption across the failure and no failure states. Since the failure state does not exist for safe sellers, we restrict both seller types to use risk transfer for the same reason. Allowing $\operatorname{prob}(N D, F)>0$ will actually strengthen our key result; we discuss this further in Robustness Section 7.1.
} 
maker. ${ }^{8}$ Finally, we assume market makers are well capitalized and risk neutral. ${ }^{9}$ Thus, when a market maker contracts with a risky seller, and so is subject to counterparty risk, competitive zero expected profit prices will generally require a selling price which is less than the price that the safe seller receives or the buyer pays. In reality, dealers can (and generally do) use both the price mechanism and collateral to mitigate the costs of counterparty risk. In the CDS market for example, Arora et al. (2009) find that collateral is the more important of the two in mitigating counterparty risk, whereas Morkoetter et al. (2012) show that there is a significant price impact of counterparty risk. We could include costly collateral in place of a price differential, or have both modeled simultaneously, without affecting our qualitative results and we choose to simply focus on prices for ease of exposition.

Atkeson et al. (2013) show, in an endogenous market formation model, that large banks tend to become dealers/market makers in OTC markets and contract for intermediation or trading purposes. In contrast, medium size banks tend to be on either the buy or sell side and contract more for hedging purposes. This is consistent with our model since hedgers are best described as risk averse (as we model buyers and sellers), and those using the contracts as an intermediary should be modeled as being less risk averse, in our case, we simply assume our market maker is risk neutral.

\subsection{Contracts}

Agents use a contingent contract to transfer consumption between states. Buyers wish to transfer consumption from the state in which no loss (ND) occurs, to that in which the loss (D) occurs. For sellers, the reverse is true. Each Buyer receives $\gamma_{B}$ in states 2 and 3 , and pays $P_{B} \gamma_{B}$ in state 1. Safe sellers pay $\gamma_{S s}$ in states 2 and 3 , and receive $P_{S s} \gamma_{S s}$ in state 1. Risky sellers pay $\gamma_{S r}$ in state 2 , and receive $P_{S r} \gamma_{S r}$ in state 1 (and pay nothing in state 3 ). We refer to $\gamma_{j}$ as the contract size and $P_{j}$ as the contract price where $j \in\{B, S s, S r\}$. All contracts are with the market

\footnotetext{
${ }^{8}$ Strictly speaking, contracts are non-exclusive given competition of market makers; i.e., a buyer or seller could split its contract over market makers as in Stephens and Thompson (2014b). When we introduce asymmetric information in Section 4, we can obtain our results even with a market maker choice and so we consider the buyers and sellers interacting with one market market for ease of exposition.

${ }^{9}$ We assume that the market maker is well capitalized for simplicity, to rule out counterparty risk of the market maker itself. We could endow our market maker with sufficient wealth such that they can never fail. Including a wealth term would only enter our analIUysis in the two efficiency sections below and would not change the results.
} 
maker who offers risk-neutral zero expected profit prices. We now formalize the buyer's problem:

$$
\max _{\gamma_{B}} \pi_{1} u\left(w-P_{B} \gamma_{B}\right)+\left(\pi_{2}+\pi_{3}\right) u\left(w-L+\gamma_{B}\right)
$$

The buyer gives up $P_{B} \gamma_{B}$ in state one (the first term) and gains $\gamma_{B}$ in states two and three (the second term). Similarly, the safe seller solves:

$$
\max _{\gamma_{S s}} \pi_{1} u\left(w+P_{S s} \gamma_{S s}\right)+\left(\pi_{2}+\pi_{3}\right) u\left(w+L-\gamma_{S s}\right)
$$

Finally, the risky seller's problem is:

$$
\max _{\gamma_{S r}} \pi_{1} u\left(w+P_{S r} \gamma_{S r}\right)+\pi_{2} u\left(w+L-\gamma_{S r}\right)+\pi_{3} u(0)
$$

To summarize, Figure 1 illustrates the payments to and from the market maker.

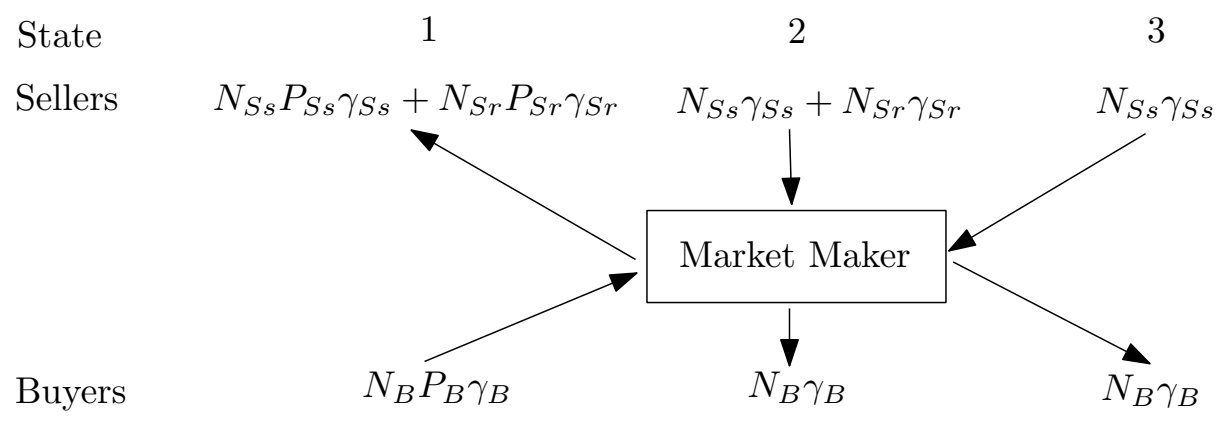

Figure 1: Payments to/from Market Maker

Note that since market maker inventory is unrestricted, buyers and sellers are not connected since the market maker absorbs all the risk. In the following section, the results will arise from the sellers interaction with the market maker. The buyers will be relevant when the link between them and the sellers is present because of limits on inventory (see Section 5).

\section{Full Information}

\subsection{Competitive Equilibrium}

As a benchmark, we consider the setting in which seller type is known. The following characterizes our equilibrium concept with full information. 
Definition 1 A full information competitive equilibrium is a set of prices $\left\{P_{B}, P_{S s}, P_{S r}\right\}$ and contract choices $\left\{\gamma_{B}, \gamma_{S s}, \gamma_{S r}\right\}$ such that

i. Given $\left\{P_{B}, P_{S s}, P_{S r}\right\}$, buyers and sellers choose $\left\{\gamma_{B}, \gamma_{S s}, \gamma_{S r}\right\}$ to maximize expected utility defined in (1), (2) and (3).

ii. $\left\{P_{B}, P_{S s}, P_{S r}\right\}$ are determined by Bertrand competition between market makers, with every buyer and seller.

In this environment we can think of two seller markets: one with safe sellers and another with risky. The market maker offers the same price to all buyers since they are homogenous. Since it is risk neutral and competitive, Bertrand competition implies that the market maker sells protection to buyers at prices which yield zero profit in expectation, so that $P_{B}=\left(\pi_{2}+\pi_{3}\right) / \pi_{1}$. Likewise, the market maker simultaneously contracts at zero expected profit from safe sellers for $P_{S s}=\left(\pi_{2}+\pi_{3}\right) / \pi_{1}$ and from risky sellers for $P_{S r}=\pi_{2} / \pi_{1}$. Lemma 1 describes consumption in the competitive equilibrium.

Lemma 1 In the full information competitive equilibrium, buyers and safe sellers perfectly smooth consumption over all states, and risky sellers over states 1 and 2. Equilibrium contract sizes are $\gamma_{B}^{*}=\gamma_{S s}^{*}=\pi_{1} L, \gamma_{S r}^{*}=\pi_{1} L /\left(\pi_{1}+\pi_{2}\right)$.

Proof. See Appendix.

As one would expect, risk-averse agents fully smooth consumption since they are able to shed risk with a risk neutral market maker offering zero-profit prices. With perfect smoothing, consumption for each type is the same in each state and denoted $\bar{c}_{B}=w-L\left(1-\pi_{1}\right), \bar{c}_{S s}=w+L\left(1-\pi_{1}\right)$ and $\bar{c}_{S r}=w+L\left(\pi_{2} /\left(\pi_{1}+\pi_{2}\right)\right)$, except for the risky seller's failure state (state 3 ) in which they consume nothing.

\subsection{Efficiency and Welfare with Full Information}

To study the efficiency properties of our environment, we characterize a standard Pareto problem. Individuals are indexed by $k \in\{B, S s, S r, M\}$, where $B$ represents the buyer, $S s$ the safe sellers, $S r$ the risky sellers, and $M$ the market maker. Let $\theta_{k} \in[0,1]$ represent a Pareto weight 
on each type of individual, normalized such that $\sum_{k} \theta_{k}=1$. Denote consumption by the vector $c_{i}=\left(c_{i}^{B}, c_{i}^{S s}, c_{i}^{S r}, c_{i}^{M}\right)$, where $i$ is the state. The first-best Pareto frontier is the solution to the following social planner's problem:

$$
\begin{array}{r}
\max _{c_{i}} W=\theta_{S r} N_{S r}\left[\pi_{1} u\left(c_{1}^{S r}\right)+\pi_{2} u\left(c_{2}^{S r}\right)+\pi_{3} u(0)\right]+\theta_{S s} N_{S s}\left[\pi_{1} u\left(c_{1}^{S s}\right)+\pi_{2} u\left(c_{2}^{S s}\right)+\pi_{3} u\left(c_{3}^{S s}\right)\right] \\
+\theta_{B} N_{B}\left[\pi_{1} u\left(c_{1}^{B}\right)+\pi_{2} u\left(c_{2}^{B}\right)+\pi_{3} u\left(c_{3}^{B}\right)\right]+\theta_{M}\left[\pi_{1} c_{1}^{M}+\pi_{2} c_{2}^{M}+\pi_{3} c_{3}^{M}\right]
\end{array}
$$

subject to

$$
\begin{array}{r}
N_{B} c_{1}^{B}+N_{S r} c_{1}^{S r}+N_{S s} c_{1}^{S s}+c_{1}^{M} \leq w\left(N_{B}+N_{S r}+N_{S s}\right) \\
N_{B} c_{2}^{B}+N_{S r} c_{2}^{S r}+N_{S s} c_{2}^{S s}+c_{2}^{M} \leq w\left(N_{B}+N_{S r}+N_{S s}\right)+L\left(N_{S r}+N_{S s}-N_{B}\right) \\
N_{B} c_{3}^{B}+N_{S s} c_{3}^{S s}+c_{3}^{M} \leq w\left(N_{B}+N_{S s}\right)+L\left(N_{S s}-N_{B}\right),
\end{array}
$$

where $F A_{1}, F A_{2}$ and $F A_{3}$ ensure allocations are feasible; namely that total consumption in a given state must be less than or equal to the total endowment in that state. Importantly, $F A_{3}$ shows that in the failure state of the risky seller (state 3), the consumption of the risky seller is forced to be zero to correspond with the assumption in the competitive market environment that no hedge for this risk exists. ${ }^{10}$

Lemma 2 The full information competitive equilibrium is efficient.

Proof. See Appendix.

As in the competitive equilibrium, the first order conditions of the Pareto problem characterize a set of social optima in which buyers and both seller types fully smooth consumption across all states (with the exception of the failure state for risky sellers).

\footnotetext{
${ }^{10}$ If a planner could hedge this state of the world, then it is obvious that the market outcome will be inefficient. Thus, we impose this restriction on the planner as well so that we can focus on the inefficiency due to asymmetric information in Section 4.2.
} 


\section{Asymmetric information}

\subsection{Competitive Equilibrium}

We now consider the setting in which information regarding seller failure risk is private, and the market maker cannot directly observe the contract size. ${ }^{11}$ All other variables remain common knowledge. To analyze the set of equilibria in this environment we need to characterize the beliefs of the market maker, which determine prices. Sellers cannot simply tell the market maker their type (i.e., there is cheap-talk) and thus risky sellers can pool with safe sellers and receive a higher price than if they were revealed as risky. Despite this, risky sellers may knowingly reveal themselves as risky through their risk transfer decision (why they may wish to do this is discussed below). Accordingly, the market maker chooses two prices; one for revealed risky sellers and the other for what we refer to as pooled sellers, who may be either risky or safe. Denote the fraction of risky sellers that reveal their type by $\rho \in[0,1]$, and the probability of a seller being safe in the pool by $\phi$. Although complete information regarding positions is not generally available when contracts are written, as discussed in Section 1 we assume that dealers can identify whether a counterparty has a position above some threshold, i.e., is a "large trader". Denote this threshold $\bar{\gamma}$, which is assumed to be an exogenous positive constant. Importantly, since market players do not learn the contract size, only that a player is above some threshold, a market maker will not offer a complete menu of contracts (as in Rothschild and Stiglitz (1976) for example). This corresponds to what we observe in real world OTC markets, since we do not observe dealers offering a full menu of contracts to buyers and sellers as seen in traditional insurance market. The way in which type information is revealed is important to the strategic behavior of risky sellers. In particular, we assume that market makers know that risky sellers wish to sell more at any given price than their safe counterparts. ${ }^{12}$ Thus, it is plausible that type information is conveyed when the contract size is above that which is optimal for safe sellers.

Consider a price $\hat{P}$ with corresponding $\gamma_{S s}^{*}(\hat{P})$ and $\gamma_{S r}^{*}(\hat{P})$, defined by $(2)$ and $(3)$ respectively.

\footnotetext{
${ }^{11}$ This is to be distinguished from a potential asymmetric information problem on the underlying risk as considered in, among others, Acharya and Johnson (2007) and Stephens and Thompson (2014b). We model only one type of underlying risk, so that we can clearly elucidate the problem of asymmetric information on counterparty risk.

${ }^{12}$ This can be easily shown by inspecting the first order conditions from the safe and risky seller problems (2) and (3).
} 
Three cases are possible:

i. $\gamma_{S s}^{*}(\hat{P})<\gamma_{S r}^{*}(\hat{P})<\bar{\gamma}$

ii. $\gamma_{S s}^{*}(\hat{P}) \leq \bar{\gamma}<\gamma_{S r}^{*}(\hat{P})$.

iii. $\bar{\gamma}<\gamma_{S s}^{*}(\hat{P})<\gamma_{S r}^{*}(\hat{P})$

Case i corresponds to an environment in which the threshold to be revealed is higher than what either type would wish to choose. As a result, information will not be revealed in equilibrium. In Case ii, a risky type may have an incentive to choose a lower contract size to receive a higher price. Case iii corresponds to an environment in which the safe type may have an incentive to choose a lower contract size to receive a higher price. Case ii is presumably the most interesting since $\bar{\gamma}$ is between what each seller type would choose in the absence of private information. While we focus on case ii, the analysis of case iii is very similar and thus we omit this for the sake of brevity (case $\mathrm{i}$ is straightforward). We assume that the market maker's beliefs satisfy the following.

Assumption 1 Assume case ii holds. Market maker beliefs satisfy:

- If the seller chooses a contract larger than $\bar{\gamma}$, beliefs are that the seller is risky.

- If the seller chooses a contract less than or equal to $\bar{\gamma}$, beliefs are not updated and reflect population averages.

Zero profit prices (which result from Bertrand competition) for the buyers and revealed risky sellers are unchanged from the case of full information, $P_{B}=\left(\pi_{2}+\pi_{3}\right) / \pi_{1}$ and $P_{S r}=\pi_{2} / \pi_{1}$ respectively. The zero (expected) profit price for the pooled sellers is denoted $\tilde{P}(\phi)$ and given by

$$
\tilde{P}(\phi)=\frac{\pi_{2}+\phi \pi_{3}}{\pi_{1}}
$$

We define the contract sizes $\gamma_{B}, \gamma_{S s}, \gamma_{S r}$ as before, however $\gamma_{S r}$ is now interpreted as the contract size of a revealing risky seller. We also define $\tilde{\gamma}_{S r} \leq \bar{\gamma}$ as the contract size of a pooling risky seller. We can now give the expression for $\phi$, the probability of a seller in the pool being safe.

$$
\phi=\frac{N_{S s} \gamma_{S s}}{N_{S s} \gamma_{S s}+(1-\rho) N_{S r} \tilde{\gamma}_{S r}} \in[\underline{\phi}, 1]
$$


Where $\underline{\phi}$ denotes the probability of a seller being safe in the pool given that all risky sellers are pooling and is given by:

$$
\underline{\phi}=\frac{N_{S s} \gamma_{S s}}{N_{S s} \gamma_{S s}+N_{S r} \tilde{\gamma}_{S r}}
$$

In equilibrium (to be defined below), if there is to be an interior solution (whereby some risky sellers reveal and some pool) a risky seller must be indifferent between revealing and pooling. Define the indirect utility of a risky seller revealing as $V^{r e v}$, and a pooling as $V^{\text {pool }}(\phi)$, when there is a proportion $\phi$ safe sellers in the pool. Let $\mathcal{V}(\phi) \equiv V^{r e v}-V^{\text {pool }}(\phi)$, which is given by:

$$
\mathcal{V}(\phi)=\pi_{1} u\left(w+P_{S r} \gamma_{S r}\right)+\pi_{2} u\left(w+L-\gamma_{S r}\right)-\left[\pi_{1} u\left(w+\tilde{P}(\phi) \tilde{\gamma}_{S r}\right)+\pi_{2} u\left(w+L-\tilde{\gamma}_{S r}\right)\right]
$$

We now augment Definition 1 to allow for asymmetric information.

Definition 2 A competitive equilibrium under asymmetric information is a set of prices $\left\{P_{B}, P_{S r}, \tilde{P}(\phi)\right\}$, contract choices $\left\{\gamma_{B}, \gamma_{S s}, \gamma_{S r}, \tilde{\gamma}_{S r}\right\}$, market maker beliefs and a pool quality $\phi$ such that:

i. Given $\left\{P_{B}, \tilde{P}(\phi), P_{S r}\right\}$, buyers, safe sellers and revealed risky sellers choose $\left\{\gamma_{B}, \gamma_{S s}, \gamma_{S r}\right\}$ to maximize expected utility defined in (1), (2) and (3). Pooling risky sellers choose $\tilde{\gamma}_{S r}$ given market maker beliefs.

ii. Market maker beliefs satisfy Assumption 1.

iii. $\left\{P_{B}, \tilde{P}(\phi), P_{S r}\right\}$ are determined by Bertrand competition among market makers, with each buyer, pooled seller, and revealed seller.

iv. $\mathcal{V}(\phi) \geq 0$ when $\phi=1, \mathcal{V}(\phi)=0$ when $\phi \in(\underline{\phi}, 1), \mathcal{V}(\phi) \leq 0$ when $\phi=\underline{\phi}$.

The safe sellers' problem is straight-forward, and is characterized by (2) at the zero-profit price implied by Bertrand competition, $P_{S s}=\tilde{P}(\phi)$. The risky sellers' problem is more complex, since they will receive price $P_{S r}$ if they reveal and choose contract size according to (3). Alternatively, they can mimic the safe types and receive the higher price $\tilde{P}(\phi)$; however, Assumption 1 implies that the contract size that they must choose cannot exceed $\bar{\gamma}$. Anything less than this amount would lower the utility of a risky seller, and anything greater would reveal their type. Therefore, 
in equilibrium $\tilde{\gamma}_{S r}=\bar{\gamma}$. We now state the main result of the section; namely that self-revelation can exist in competitive equilibrium.

Proposition 1 The competitive equilibrium with asymmetric information is characterized by $\phi^{*} \in$ $[\underline{\phi}, 1)$. Self-revelation of a subset of risky sellers, $\phi^{*} \in(\underline{\phi}, 1)$, can be supported as an equilibrium.

Proof. See Appendix.

With a standard asymmetric information problem, the "risky" type prefers to pool with the "safe" type because they receive a better price. This is the reasoning behind the classic Akerlof (1970) lemons result. In our model however, there is a second effect which works against pooling: imitating the safe seller requires the risky seller to take a contract size of $\bar{\gamma}$. As more risky sellers reveal, the quality of the pool increases and so too does the price that those who remain in the pool receive. The limiting case in which all risky sellers reveal $\left(\rho=1\right.$ so that $\left.\phi^{*}=1\right)$ is not an equilibrium, however. This case would correspond to the full information environment described in Section 3. From Lemma 1, we know that with full information the safe and risky sellers perfectly smooth consumption over states 1 and 2 (with the safe seller also smoothing over state 3 ). Since a safe seller receives a higher price, it obtains higher consumption in every state relative to a risky seller. Thus, given that no other risky sellers pool, a single risky seller could pool and receive a higher (smoothed) consumption profile. If we were to allow for a risky seller to reduce its counterparty risk through contracting (i.e., having 4 instead of 3 states), we could achieve full separation for some parameter range in which all risky sellers reveal. See Robustness Section 7.1 for a further discussion.

\subsection{Efficiency and Welfare with Asymmetric Information}

To analyze the efficiency properties of the competitive equilibrium under asymmetric information, we consider the Pareto problem described in (4), with the following additional incentive constraints.

$$
\begin{array}{r}
\pi_{1} u\left(c_{1}^{S r}\right)+\pi_{2} u\left(c_{2}^{S r}\right) \geq \pi_{1} u\left(c_{1}^{S s}\right)+\pi_{2} u\left(c_{2}^{S s}\right) \\
\pi_{1} u\left(c_{1}^{S s}\right)+\pi_{2} u\left(c_{2}^{S s}\right)+\pi_{3} u\left(c_{3}^{S s}\right) \geq \pi_{1} u\left(c_{1}^{S r}\right)+\pi_{2} u\left(c_{2}^{S r}\right)+\pi_{3} u(0)
\end{array}
$$


$I C_{1}$ ensures that the consumption bundle intended for the risky type is indeed preferred by the risky type. As in Section 3.2, to remain consistent with the assumption that no hedge for the failure risk exists, the risky type receives nothing in state 3 regardless of which consumption bundle is chosen. $I C_{2}$ ensures that the safe type prefers its own consumption bundle. Analyzing $I C_{1}$ and $I C_{2}$, it follows that if one is satisfied with equality, then the other is automatically satisfied. The solution to this problem is considered in the proof to the following lemma.

Lemma 3 The asymmetric information competitive equilibrium is constrained inefficient.

Proof. See Appendix.

The inefficiency arises because a planner can achieve full revelation by cross subsidizing between the two selling types, i.e., the planner is not restricted to earn zero expected profit in both seller markets, as is the market maker. Further, as discussed below, relative to a market maker restricted from holding an inventory, the social planner is also able to increase risk transfer since buyers are rationed in equilibrium.

Lemma 3 suggests that an implementable Pareto improving policy exists. Indeed, there are Pareto improving tax/subsidy market interventions that can be implemented. The key is that such a policy must encourage revelation either by making pooling less desirable, or revelation more desirable for the risky types. The following Proposition considers the imposition of a unit tax on pooling sellers, resulting in the after-tax price $\tilde{P}-t$.

Proposition 2 A transaction tax on pooling sellers is Pareto improving.

Proof. See Appendix.

The intuition behind this result can be seen by noting that the only net (after tax) price that can hold in equilibrium is the before-tax price. This is because the tax drives pooling risky sellers to reveal and thus makes the pool of higher quality. Since the payoff to revealing is independent of the tax, the price in the pool adjusts as risky sellers reveal so that both safe and risky sellers are just as well off with the tax than without. Although we focus on a simple tax to pooling sellers, there are other policies which would yield a similar outcome. For example, a per-unit transaction 
subsidy on all sellers would favor revelation since risky sellers supply more with revelation. In this case, an appropriate lump-sum (participation) tax could be used to finance the subsidy.

\section{Inventory}

The assumption of unlimited inventory simplifies the previous analysis; however, this is clearly not a realistic assumption, especially in the presence of counterparty risk. For example, Shachar (2012) provides evidence that such dealers are averse to holding large inventories due to counterparty risk. ${ }^{13}$ In this section, we generalize our previous results on information revelation and consider new implications of information asymmetry on the buy side of the market. We leave the reasons for inventory restrictions unmodeled, although features such as risk aversion of the market maker could be added to accomplish this; however, this would substantially complicate the analysis.

In a traditional asset market, restrictions on inventory are relatively straightforward: if a market maker cannot hold any inventory, it can only sell as much as it buys. With risk transfer contracts however, counterparty risk necessitates that the market maker bear some risk, i.e., although the market maker can earn zero expected profit from each contract ex-ante, they do not necessarily earn zero profit ex-post. To understand this, consider the case where there are no risky sellers. In this setting, the amount that sellers pay the market maker in states 2 and 3 is the same. If the market maker cannot hold an inventory, then in addition to earning zero expected profit ex-ante due to competition, it must earn zero profit ex-post, regardless of which event occurs. The total contract size that buyers request is $N_{B} \gamma_{B}^{*}$, which is paid to them in states 2 and 3 , where $\gamma_{B}^{*}$ solves (1). Sellers request $N_{S s} \gamma_{S s}^{*}$, which they would owe to the market maker in state 1 , where $\gamma_{S s}^{*}$ solves (2). Since the market maker must break even ex-post, if $N_{S s} \gamma_{S s}^{*}>N_{B} \gamma_{B}^{*}$ $\left(N_{S s} \gamma_{S s}^{*}<N_{B} \gamma_{B}^{*}\right)$, the sellers (buyers) cannot generally obtain their optimal contract size. We denote the total number of contracts that can be traded with the buyers or sellers as $\gamma_{M M}$, which is defined by $\gamma_{M M}=\min \left(N_{S s} \gamma_{S s}^{*}, N_{B} \gamma_{B}^{*}\right)$. With this contract size, the market maker will make zero profit in states 2 and 3 . To verify that the market maker makes zero profit in state 1 , we note that they must pay the sellers $\gamma_{M M} P_{S s}$, while they will receive $\gamma_{M M} P_{B}$ from the buyers. Thus, they will break even in state 1 when $P_{B}=P_{S s}$, which turn out to be precisely the prices that

\footnotetext{
${ }^{13}$ Chen et al. (2011) also consider the issue of inventory and show that when taking large positions, the dealer may hold the inventory temporarily, presumably to avoid market impact while hedging.
} 
guarantee zero expected profits ex-ante and are described further in Section 3.1. Hence, the market maker will earn zero expected profit ex-ante, as well as zero profit ex-post in all states of the world.

Now consider the addition of $N_{S r}$ risky sellers. The market maker breaks even in state 2 when

$$
\gamma_{M M}=\min \left(N_{S s} \gamma_{S s}^{*}+N_{S r} \gamma_{S r}^{*}, N_{B} \gamma_{B}^{*}\right)
$$

In state 3 however, the market maker must pay the buyers $N_{B} \gamma_{B}^{*}$, but would receive only $N_{S s} \gamma_{S s}^{*}<$ $N_{B} \gamma_{B}^{*}$ from the sellers since the risky sellers do not pay in this state. It is relatively straightforward to show that the market maker will not break even ex-post in state 1 either.

We must also consider how prices are formed through competition with limited inventory. With unlimited inventory, it was simplest to view buyers and sellers interacting with just one market maker. With limited inventory however, competition between market makers makes the potential equilibria more complicated. To avoid undue distraction, we make a simple assumption which determines prices as in the case of unlimited inventory; however, also allows us to analyze the interesting effects that limited inventory can have on buyers and sellers. We assume that the measure of market makers are limited on inventory, so that it must ration contracts to ensure that it breaks even in state $2 .{ }^{14}$ However, we assume that individual market makers can hold a "small" (i.e., infinitesimal) inventory. This assumption will lead to zero profit prices, and as will be demonstrated, can lead to rationing of contracts. In Robustness section 7.2 , we discuss how the results of this section will come through in an environment in which prices can adjust and eliminate rationing. Note that if contracts are rationed, we assume that in the presence of an excess of supply (demand), the market maker rations the available contracts among the sellers (buyers) equally.

\subsection{Limited Inventory with Full Information}

When the inventory of the market maker is limited, the markets may not clear since either buyers or sellers may not be able to obtain their optimal contract size. This can occur because the market maker must make zero expected profit on each contract and so prices cannot adjust to eliminate excess demand or supply. First, we extend Definition 1 to account for inventory restrictions.

\footnotetext{
${ }^{14}$ Similar results can be derived if they instead break even ex-post in states 1 or 3
} 
Definition 3 A full information competitive equilibrium with limited inventory is that of Definition 1, with the following additional condition:

iii. Market maker contracts satisfy (9).

When the market maker has inventory restrictions, there will generally be some rationing. The following lemma summarizes.

Lemma 4 The full information competitive equilibrium with limited inventory is identical to that with inventory if and only if

$$
N_{B}=N_{S s}+\frac{N_{S r}}{\pi_{1}+\pi_{2}}
$$

Proof. See Appendix.

Thus the full information competitive equilibrium is efficient when the market maker is unrestricted in taking an inventory (Lemma 2), or when the market maker is restricted on inventory and equation (10) is satisfied. When the market maker is restricted on inventory and markets do not clear at zero expected profit prices (i.e., when (10) is not satisfied), full consumption smoothing is not attained for either the buyers or sellers and the competitive equilibrium is inefficient. To aid in the analysis of asymmetric information below, we make the following assumption.

Assumption 2 When the market maker is restricted from taking inventory, equation (10) is assumed to hold.

Thus, we will focus on the case in which the competitive equilibrium is efficient under full information regardless of inventory restrictions. While not important for the results in the paper, this assumption highlights the inefficiency from asymmetric information by starting with an efficient equilibrium under full information.

\subsection{Limited Inventory with Asymmetric Information}

With no restrictions on inventory, buyers in our model are unaffected by the presence of asymmetric information on the sell side of the market. This is not necessarily the case however, when the 
inventory of the market maker is restricted. We begin by determining how asymmetric information affects the total number of contracts that sellers and buyers trade. In particular, how sellers react to changes in prices will determine whether the total number of contracts increases or decreases under asymmetric information. Defining $\rho^{*}$ and $\phi^{*}$ as the corresponding equilibrium values of $\rho$ and $\phi$, the total contract size requested from sellers under asymmetric information is:

$$
N_{S s} \gamma_{S s}\left(\phi^{*}\right)+\left(1-\rho^{*}\right) N_{S r} \bar{\gamma}+\rho^{*} N_{S r} \gamma_{S r}
$$

The first term is the total requested contract size of the safe sellers in the pool, the second term is the total requested contract size of the pooling risky sellers, while the third term represents the total requested contract size of the revealing risky sellers. Note that $\gamma_{S s}\left(\phi^{*}\right)$ is the optimal contract size of the safe sellers with asymmetric information. Total requested contract size of the sellers with full information is $N_{S s} \gamma_{S s}(\phi=1)+N_{S r} \gamma_{S r}$, where $\gamma_{S s}(\phi=1)$ is the optimal contract size of the safe sellers under full information. We denote the difference between the total requested contract size under full and asymmetric information as

$$
\Delta=N_{S r}\left(1-\rho^{*}\right)\left[\gamma_{S r}-\bar{\gamma}\right]+N_{S s}\left[\gamma_{S s}(\phi=1)-\gamma_{S s}\left(\phi^{*}\right)\right]
$$

The first term represents the change in contract size for risky sellers who pool, whereas the second term represents the change in contract size of safe sellers. Whether the total number of requested contracts increases or decreases under asymmetric information (i.e., whether $\Delta \lessgtr 0$ ) cannot be ascertained at the current level of generality. However, when the safe sellers' contract supply curve is upward sloping, so that requested contract size increases in price, then total requested contracts decrease as a result of the information friction.

Lemma 5 If $\frac{d \gamma_{S s}}{d P_{S s}} \geq 0$, then $\Delta>0$.

Proof. See Appendix.

The intuition behind this result is that safe sellers decrease contract size in the pool relative to the full information case and risky sellers also decrease contract size when pooling versus when they are revealed, thus total contract size falls. In a standard asset market, appropriate assumptions 
on utility are often made to ensure that supply is upward sloping. For the risk transfer contract modeled here, a higher price is interpreted as state contingent: more consumption can be obtained in state 1 relative to state 2 for a fixed contract size. In this environment, we can ensure that supply is upward sloping with appropriate restrictions on risk aversion and/or the size of the loss, as is formalized in the following lemma.

Lemma $6 \frac{d \gamma_{S s}}{d P_{S s}} \geq(<) 0$ when $\frac{-u^{\prime \prime}\left(w+P_{S s} \gamma_{S s}\right)\left(w+P_{S s} \gamma_{S s}\right)}{u^{\prime}\left(w+P_{S s} \gamma_{S s}\right)} \leq(>) 1+\frac{w}{P_{S s} \gamma_{S s}}$.

Proof. See Appendix.

The left hand side of this expression is the safe sellers' measure of relative risk aversion (evaluated at state 1$)$. We can bound the right hand side using $L \geq\left(1+P_{j}\right) \gamma_{j}$, ruling out "overinsurance", which cannot occur in the equilibrium in which we focus. ${ }^{15}$ Thus $1+w / P_{S s} \gamma_{S s} \geq 1+w / L$. To simplify the analysis, we make the following assumption which is assumed to hold throughout the remainder of this section.

Assumption 3 When inventory is limited, we assume the coefficient of relative risk aversion is less than $(w+L) / L$.

This ensures that safe seller supply is increasing in price (and thus $\Delta>0$ ) and requires that relative risk aversion is sufficiently low and/or the size of the loss being insured is a relatively small portion of the sellers portfolio. It is important to note that $d \gamma_{S s} / d P_{S s} \geq 0$ is merely a sufficient condition to ensure $\Delta>0$, and that $d \gamma_{S s} / d P_{S s}<0$ can hold and $\Delta>0$ still obtain. To understand why, when $d \gamma_{S s} / d P_{S s}<0, \gamma_{S s}$ increases with asymmetric information so that the safe sellers have an increased contract size; however, this can be dominated by a drop in contract size of the risky sellers. ${ }^{16}$ We now extend Definition 1 to account for inventory restrictions and then give the main result as a simple extension of Proposition 1.

\footnotetext{
${ }^{15}$ In the equilibrium defined below, the price faced by the safe seller is never better than that which is actuarially fair and thus they will not contract beyond full smoothing of consumption (nor is a negative choice of $\gamma_{S s}$ optimal).

${ }^{16}$ Note that the analysis of the problem if $\Delta<0$ is similar to what we present here; however, the equilibrium is somewhat more complicated. Importantly, the self-revelation result to be detailed below can still obtain.
} 
Definition 4 A competitive equilibrium under asymmetric information with limited inventory is that of Definition 2, with the following additional condition:

v. $\gamma_{M M}=\min \left(N_{S s} \gamma_{S s}^{*}+(1-\rho) N_{S r} \bar{\gamma}+\rho^{*} N_{S r} \gamma_{S r}^{*}, N_{B} \gamma_{B}^{*}\right)$, so that the market maker breakseven in state 2.

Corollary 1 The competitive equilibrium with asymmetric information and limited inventory is unique and characterized by $\phi^{*} \in[\underline{\phi}, 1)$. Self-revelation of a subset of risky sellers, $\phi^{*} \in(\underline{\phi}, 1)$, can be supported as an equilibrium.

The proof of this case is identical to that for Proposition 1 since there is only rationing on the buy side of the market, and thus the sell side remains unchanged.

As is true in a standard model with asymmetric information, the low risk type (safe seller in our context) is unambiguously worse off as a result of the information friction. On the other hand, when $\phi^{*}>\underline{\phi}$, the risky seller receives the same utility with or without asymmetric information. To understand this, note that when $\phi^{*}>\underline{\phi}$, there is revelation in equilibrium. The risky sellers that reveal receive the same payoffs as with full information. Since the asymmetric information competitive equilibrium requires that a risky seller is indifferent between pooling and revealing, risky sellers' that pool must have the same expected utility as the full information case.

On the buy side of the market, when the market maker can take unlimited inventory, buyers always obtain the contract size they request since the information friction is irrelevant from their perspective. With limited inventory however, the number of contracts to which the buyers can obtain is affected by asymmetric information on the sell side. The following result follows easily from Corollary 1.

Corollary 2 With restricted inventory, buyers are worse off due to the existence of asymmetric information.

This follows simply from the reduction in total requested contract size of the sellers under asymmetric information. As a result, the market maker must decrease the contract size available to the buyers below that which is optimal. Hence, buyers are worse off under asymmetric information than under full information. In light of this, we note that Proposition 2 can be extended to the case with restricted inventory. The same argument applies, but in addition the increase in supply 
unambiguously increases buyer utility and so the tax achieves a Pareto improvement even in the absence of any redistribution.

\section{Concluding Remarks}

We model an intermediated/dealer financial market for risk transfer under counterparty risk. When sellers have asymmetric information on their own counterparty risk, in contrast to the classic Akerlof (1970) lemons result, we show that some risky sellers may reveal themselves, allowing a separating equilibrium to exist purely by market forces. The competitive equilibrium is shown to be information constrained inefficient due to insufficient information revelation and a simple transaction tax on pooling counterparties is shown to be Pareto improving.

Underlying the results of this paper is the assumption that the market maker is able to distinguish "large traders". If the positions of buyers and sellers are completely hidden, then a separating equilibrium of the type described in Proposition 1 is not possible. One of the proposed outcomes of centralized clearing is that data can be collected on positions of its members. A potential benefit claimed by regulators is that they can use this data to deduce whether risks are gathering in particular parts of the financial system, or with specific financial institutions. We contend that because this information is used to identify parties that take large positions, it can actually promote transparency through a previously unstudied mechanism; market players may willfully reveal their own risk. The specific mechanism whereby a central counterparty (CCP) pools counterparty risk is beyond the scope of the paper. ${ }^{17}$ However, we note that since the CCP is the eventual counterparty in every transaction, the penalty to a revealed risky seller need not come only in the form of a lower price as we have modeled here. The CCP itself could set higher participation charges on the revealed risky seller, for example, through collateral requirements or contributions to a default pool. The separating mechanism that we describe in the paper can then follow wherein a subset of risky sellers reveal and obtain larger contract sizes, but are penalized by the CCP to account for the increased counterparty risk to which they pose.

\footnotetext{
${ }^{17}$ For a general discussion on CCPs see Bliss and Steigerwald (2006).
} 


\section{Robustness}

\subsection{Correlation of asset and risky seller failure}

We assumed for simplicity that $\operatorname{prob}(N D, F)=0$. This was done to make our risky and safe sellers as homogenous as possible while still obtaining our results. When this assumption is relaxed, our separation result becomes stronger; in particular, full separation wherein every risky seller reveals its type can be supported as an equilibrium. To see this, define 4 states of the world: $\tilde{\pi_{1}}=\operatorname{prob}(N D, N F), \tilde{\pi_{2}}=\operatorname{prob}(D, N F), \tilde{\pi_{3}}=\operatorname{prob}(D, F), \tilde{\pi_{4}}=\operatorname{prob}(N D, F)$. Note that $\pi_{1}=\tilde{\pi_{1}}+\tilde{\pi_{4}}, \pi_{2}=\tilde{\pi_{2}}$ and $\pi_{3}=\tilde{\pi_{3}}$. Consider the problem of a risky seller under full information:

$$
\max _{\gamma_{S r}} \tilde{\pi_{1}} u\left(w+P_{S r} \gamma_{S r}\right)+\tilde{\pi_{2}} u\left(w+L-\gamma_{S r}\right)+\tilde{\pi_{3}} u(0)+\tilde{\pi_{4}} u\left(P_{S r} \gamma_{S r}\right)
$$

This leads to the optimal $\tilde{\gamma}_{S r}^{*}$ defined implicitly by:

$$
u^{\prime}\left(w+L-\tilde{\gamma}_{S r}^{*}\right)=\frac{\tilde{\pi_{1}} P_{S r}}{\tilde{\pi}_{2}} u^{\prime}\left(w+P_{S r} \tilde{\gamma}_{S r}^{*}\right)+\frac{\tilde{\pi_{4}} P_{S r}}{\tilde{\pi_{2}}} u^{\prime}\left(P_{S r} \tilde{\gamma}_{S r}^{*}\right)
$$

Comparing (14) to the first order condition from (3), it is apparent that risky sellers supply more for any given price in this relaxed setting. Now consider the effect that this has under asymmetric information. Proposition 1 showed that some risky sellers reveal to obtain a larger contract size. The difference between the contract size in the pool, versus that which is supplied with revelation is integral. When $\operatorname{prob}(N D, F)>0$, risky sellers desire a larger contract size, making the incentive to reveal even stronger. Furthermore, with $\operatorname{prob}(N D, F)>0$, the risky sellers are able to smooth over their failure state which they were not able to do when $\operatorname{prob}(N D, F)=0$. Thus, it is possible that all risky sellers reveal since they may not obtain sufficient consumption in their failure state by pooling.

\subsection{Rationing}

The simplifying assumptions made in Section 5 allow us to consider the implications of inventory restrictions in a setting most like that of Sections 2-4. That contracts are rationed in our equilibrium is not vital to the results. In an environment in which prices may adjust to clear markets, similar 
results will obtain. In particular, continue to assume market makers are competitive and offer a set of zero expected-profit prices; however, now assume that market makers cannot hold a "small" inventory. In this case, contracts may not have zero profit prices in each market as in the paper. Although more tedious than the analysis above, the zero expected profit assumption, the inventory restriction, and the market-clearing condition can be used to characterize equilibrium prices.

In such an environment, buyers utility will again be reduced due to asymmetric information. This occurs not through rationing of contracts as in the paper, but through the price mechanism. Whereas the decrease in supply of contracts due to asymmetric information resulted in rationing for buyers, now the market maker lowers the price for the buyers, in addition to increasing the price to sellers. In other words, inventory restrictions imply that a decrease in contracts supplied requires a decrease in contracts demanded. To satisfy the market maker's zero expected profit condition, the buyers price must increase to compensate for the increased price required to attract more supply. In this environment, revelation as per Corollary 1 can still exist since the benefit to a risky seller of revealing remains, and importantly, the key inefficiency result still obtains since pooling risky sellers do not consider the implications of their behavior on safe sellers or buyers, as is the case in Lemma 3. 


\section{Appendix}

\section{Proof of Lemma 1}

At zero expected profit prices, the first order conditions for problems (1), (2), and (3) are

$$
\begin{array}{r}
u^{\prime}\left(w-L+\gamma_{B}^{*}\right)=u^{\prime}\left(w-\frac{\pi_{2}+\pi_{3}}{\pi_{1}} \gamma_{B}^{*}\right) \\
u^{\prime}\left(w+L-\gamma_{S s}^{*}\right)=u^{\prime}\left(w+\frac{\pi_{2}+\pi_{3}}{\pi_{1}} \gamma_{S s}^{*}\right) \\
u^{\prime}\left(w+L-\gamma_{S r}^{*}\right)=u^{\prime}\left(w+\frac{\pi_{2}}{\pi_{1}} \gamma_{S r}^{*}\right)
\end{array}
$$

respectively. Since $u^{\prime}>0,(15)$ and (16) imply that both buyers and safe sellers smooth consumption over all states, while (17) implies that the risky seller smoothes consumption over states 1 and 2 . Further; $\gamma_{B}^{*}=\gamma_{S s}^{*}=\pi_{1} L, \gamma_{S r}^{*}=\pi_{1} L /\left(\pi_{1}+\pi_{2}\right)$.

\section{Proof of Lemma 2}

Define the Lagrange multiplier on $F A_{1}$ as $\lambda_{1}, F A_{2}$ as $\lambda_{2}$, and $F A_{3}$ as $\lambda_{3}$. The first-order conditions for the Pareto problem (4) can be written as follows.

$$
\begin{array}{r}
\theta_{S r} u^{\prime}\left(c_{i}^{S r}\right)=\lambda_{i} / \pi_{i} \text { for } i \in\{1,2\} \\
\theta_{S s} u^{\prime}\left(c_{i}^{S s}\right)=\lambda_{i} / \pi_{i} \text { for } i \in\{1,2,3\} \\
\theta_{B} u^{\prime}\left(c_{i}^{B}\right)=\lambda_{i} / \pi_{i} \text { for } i \in\{1,2,3\} \\
\theta_{M}=\lambda_{i} / \pi_{i} \text { for } i \in\{1,2,3\}
\end{array}
$$

Since $u^{\prime}\left(c_{i}^{k}\right)=\theta_{M} / \theta_{k}$ for $k \in\{B, S s, S r\}$, consumption is constant across states (except the failure state at the risky seller). To show that the competitive equilibrium is efficient, we characterize the set of Pareto weights that correspond to this allocation. Consumption in each state of the competitive equilibrium is $\bar{c}_{B}=w-L\left(1-\pi_{1}\right), \bar{c}_{S s}=w+L\left(1-\pi_{1}\right)$ and $\bar{c}_{S r}=w+L\left(\pi_{2} /\left(\pi_{1}+\right.\right.$ $\left.\pi_{2}\right)$ ). Combining $u^{\prime}\left(c_{i}^{k}\right)=\theta_{M} / \theta_{k}$ and $\sum_{k} \theta_{k}=1$, the Pareto weights which yield the competitive 
equilibrium allocation are:

$$
\theta_{M}=\frac{1}{1+\sum_{k \neq M}\left(\frac{1}{u^{\prime}\left(\bar{c}_{k}\right)}\right)}, \text { and } \theta_{k \neq M}=\frac{\theta_{M}}{u^{\prime}\left(\bar{c}_{k}\right)}
$$

\section{Proof of Proposition 1}

Step 1 proves that there exists a $\phi^{*} \in[\phi, 1)$, and step 2 shows that there exists parameters for which $\phi^{*}>\underline{\phi}$.

Step 1

Suppose $\phi^{*}=1$. Then $\mathcal{V}(1) \geq 0$. Using $\gamma_{S r}^{*}=\pi_{1} L /\left(\pi_{1}+\pi_{2}\right), \gamma_{S s}^{*}=\pi_{1} L$ from Lemma 1, and $\tilde{\gamma}_{S r}=\bar{\gamma}$, we obtain:

$$
\begin{array}{r}
\left(\pi_{1}+\pi_{2}\right) u\left(w+L\left(1-\frac{\pi_{1}}{\pi_{1}+\pi_{2}}\right)\right) \geq \pi_{1} u\left(w+\left(\frac{\pi_{2}+\pi_{3}}{\pi_{1}}\right) \bar{\gamma}\right)+\pi_{2} u(w+L-\bar{\gamma}) \\
\geq\left(\pi_{1}+\pi_{2}\right) u\left(w+L\left(1-\pi_{1}\right)\right) \geq 0 \\
\Rightarrow \pi_{1}+\pi_{2} \geq 1 .
\end{array}
$$

The second inequality follows by putting $\gamma_{S s}^{*}$ in place of $\bar{\gamma}$ since we know that $\bar{\gamma} \geq \gamma_{S s}^{*}$. The last inequality provides a contradiction since $\pi_{3}>0$ and $\pi_{1}+\pi_{2}+\pi_{3}=1$. Hence, $\phi^{*}<1$. If $\mathcal{V}(\underline{\phi}) \leq 0$, then $\phi^{*}=\phi$. If $\mathcal{V}(\phi)>0$, then, since $\mathcal{V}$ is continuous, by the intermediate value theorem there exists a $\phi^{*} \in(\phi, 1)$ such that $\mathcal{V}\left(\phi^{*}\right)=0$.

Step 2

Let all risky sellers pool and the relative number of risky to safe sellers be arbitrarily large, so that $\phi=\underline{\phi} \rightarrow 0$ and $\tilde{P} \rightarrow P_{S r}$. Then, it must be that at least one risky seller would wish to reveal, i.e., $\phi^{*}>\underline{\phi}$, since $\gamma_{S r}$ is the optimal contract size (and $\bar{\gamma}$ is suboptimal) given $P_{S r}$. 
Proof of Lemma 3

Substituting $\tilde{P}(\phi)=\left(\pi_{2}+\phi \pi_{3}\right) / \pi_{1}$ into the first order condition for problem (2) yields

$$
u^{\prime}\left(w+L-\gamma_{S s}^{*}\right)=\frac{\pi_{2}+\phi^{*} \pi_{3}}{\pi_{2}+\pi_{3}} u^{\prime}\left(w+P_{S s} \gamma_{S s}^{*}\right)
$$

Since $\phi^{*}<1$ (Proposition 1), the safe seller does not smooth consumption over states 1 and 2 in the competitive equilibrium. Turning to the social planning problem, the first-order condition for the market maker is identical to the full information problem (where again, the Lagrange multipliers on the constraints are represented by $\lambda_{i}$ ),

$$
\theta_{M}=\lambda_{i} / \pi_{i} \text { for } i \in\{1,2,3\}
$$

Using (25), the first-order conditions for the sellers are

$$
\begin{array}{r}
u^{\prime}\left(c_{i}^{S r}\right)=\frac{\theta_{M} N_{S r}}{\theta_{S r} N_{S r}+\alpha_{1}-\alpha_{2}}, \text { for } i \in\{1,2\} \\
u^{\prime}\left(c_{1}^{S s}\right)=u^{\prime}\left(c_{2}^{S s}\right)=\frac{\theta_{M} N_{S s}}{\theta_{S s} N_{S s}+\alpha_{2}-\alpha_{1}}, u^{\prime}\left(c_{3}^{S s}\right)=\frac{\theta_{M} N_{S s}}{\theta_{S s} N_{S s}+\alpha_{2}} .
\end{array}
$$

Where $\alpha_{1}$ and $\alpha_{2}$ are the Lagrange multipliers for $\left(I C_{1}\right)$ and $\left(I C_{2}\right)$ respectively. Since the allocation in the Pareto problem has consumption smoothing over states 1 and 2 for the safe seller, while the competitive equilibrium does not, the competitive equilibrium cannot be on the Pareto frontier and so is constrained inefficient.

\section{Proof of Proposition 2}

We first show that a small increase in $t$ has no impact on the utility of safe sellers. The competition assumption implies that the tax on contracts in the pooled market falls entirely on the sellers, so that the pooled price after tax is

$$
\tilde{P}(t)=\frac{\pi_{2}+\pi_{3} \phi(t)}{\pi_{1}}-t
$$


The safe seller problem is

$$
\max _{\gamma_{S s}} \pi_{1} u\left(w+\tilde{P}(t) \gamma_{S s}\right)+\left(\pi_{2}+\pi_{3}\right) u\left(w+L-\gamma_{S s}\right)
$$

which yields the optimal supply $\gamma(\tilde{P}(t), w, L)$ and indirect utility $v(\tilde{P}(t), w, L)$. Differentiating indirect utility (and using the envelope theorem) with respect to $t$ yields:

$$
\frac{d v}{d t}=\pi_{1} u^{\prime}\left(w+\tilde{P} \gamma_{S s}\right) \gamma_{S s} \frac{d \tilde{P}}{d t}
$$

We differentiate the equilibrium condition (8) with respect to the tax, which yields

$$
0=-\pi_{1} u^{\prime}(w+\tilde{P} \bar{\gamma}) \bar{\gamma} \frac{d \tilde{P}}{d t}
$$

Thus $d \tilde{P} / d t=0$, and so $d v / d t=0$ from (30). Therefore, risky sellers that reveal are indifferent to the tax since their utility is independent of a tax on the pool. Since $d \tilde{P} / d t=0$, risky sellers who pool are also unaffected by the tax. Therefore, a lump sum redistribution of the tax to the buyer for example, would yield a Pareto improvement.

\section{Proof of Lemma 4}

There is no rationing of contracts when $N_{B} \gamma_{B}^{*}=N_{S s} \gamma_{S s}^{*}+N_{S r} \gamma_{S r}^{*}$, in which case the allocation is identical to that without inventory restrictions. At zero expected profit prices, $\gamma_{B}^{*}=\gamma_{S s}^{*}=\pi_{1} L$, $\gamma_{S r}^{*}=\pi_{1} L /\left(\pi_{1}+\pi_{2}\right)$, and $N_{B} \gamma_{B}^{*}=N_{S s} \gamma_{S s}^{*}+N_{S r} \gamma_{S r}^{*}$ reduces to (10).

\section{Proof of Lemma 5}

The first term in (12) is positive by assumption. The second term is non-negative since $\gamma_{S s}(\phi=$ 1) $>\gamma_{S s}\left(\phi=\phi^{*}\right)$ when $d \gamma_{S s} / d P_{S s}>0$ and $\gamma_{S s}(\phi=1)=\gamma_{S s}\left(\phi=\phi^{*}\right)$ when $d \gamma_{S s} / d P_{S s}=0$. 


\section{Proof of Lemma 6}

Differentiating the first order condition to (2) with respect to $P_{S s}$ and re-arranging yields:

$$
\frac{d \gamma_{S s}}{d P_{S s}}=\frac{-\pi_{1}\left(u^{\prime}\left(w+P_{S s} \gamma_{S s}\right)+P_{S s} \gamma_{S s} u^{\prime \prime}\left(w+P_{S s} \gamma_{S s}\right)\right)}{\left(\pi_{2}+\pi_{3}\right) u^{\prime \prime}\left(w+L-\gamma_{S s}\right)+\pi_{1} P_{S s}^{2} u^{\prime \prime}\left(w+P_{S s} \gamma_{S s}\right)}
$$

The denominator of (32) is negative, and so the sign of $d \gamma_{S s} / d P_{S s}$ is determined by the numerator. Thus, $d \gamma_{S s} / d P_{S s}$ is non-negative (strictly negative) when:

$$
\frac{-u^{\prime \prime}\left(w+P_{S s} \gamma_{S s}\right)}{u^{\prime}\left(w+P_{S s} \gamma_{S s}\right)} \leq(>) \frac{1}{P_{S s} \gamma_{S s}}
$$

Multiplying both sides by $\left(w+P_{S s} \gamma_{S s}\right)$ yields the condition in Lemma 6 . 


\section{References}

Acharya, Viral and Alberto Bisin, "Counterparty Risk Externality: Centralized Versus Over-theCounter Markets," Journal of Economic Theory, 149 (2014), 153-182.

Acharya, Viral and Timothy Johnson, "Insider Trading in Credit Derivatives," Journal of Financial Economics, 84 (2007), 110-141.

Akerlof, George A., "The Market for 'Lemons': Quality Uncertainty and the Market Mechanism," Quarterly Journal of Economics, 84 (1970), 488-500.

Ales, Laurence and Pricila Maziero, "Adverse Selection and Non-Exclusive Contracts," Wharton School Working Paper, (2014).

Arora, Navneet, Priank Gandhi, and Francis A. Longstaff, "Counterparty Credit Risk and The Credit Default Swap Market," UCLA Working Paper, (2009).

Atkeson, Andrew G., Andrea L. Eisfeldt, and Pierre-Olivier Weill, "The Market for OTC Derivatives," UCLA Working Paper, (2013).

Attar, Andrea, Thomas Mariotti, and François Salanié, "Nonexclusive Competition in the Market for Lemons," Econometrica, 79 (2011), 1869-1918.

Biais, Bruno, Florian Heider, and Marie Hoerova, "Risk-sharing or Risk-taking? Counterparty Risk, Incentives and Margins," Forthcoming, Journal of Finance, (2014).

Bliss, Robert R. and Robert Steigerwald, "Derivatives Clearing and Settlement: a Comparison of Central Counterparties and Alternative Structures," Economic Perspectives, 4Q (2006), 2229.

Chen, Kathryn, Michael Fleming, John Jackson, Ada Li, and Asani Sarkar, "An Analysis of CDS Transactions: Implications for Public Reporting," Federal Reserve Bank of New York Staff Reports (2011).

Easley, David and Maureen O'Hara, "Price, Trade Size, and Information in Securities Markets," Journal of Financial Economics, 19 (1987), 69-90. 
Leitner, Yaron, "Inducing Agents to Report Hidden Trades: A Theory of an Intermediary," Review of Finance, 16 (2012), 1013-1042.

Morkoetter, Stefal, Johanna Pleus, and Simone Westerfeld, "The impact of counterparty risk on credit default swap pricing dynamics," Journal of Credit Risk, 8 (2012), 63-88.

Rothschild, Michael and Joseph Stiglitz, "Equilibrium in Competitive Insurance Markets: An Essay on the Economics of Imperfect Information," Quarterly Journal of Economics, 125 (1976), 629-649.

Seppi, Duane J., "Equilibrium Block Trading and Asymmetric Information," Journal of Finance, 45 (1990), 73-94.

Shachar, Or, "Exposing the Exposed: Intermediation Capacity in the Credit Default Swap Market," Working Paper, Stern School of Business, New York University, (2012).

Stephens, Eric and James R. Thompson, "CDS as Insurance: Leaky Lifeboats in Stormy Seas," Journal of Financial Intermediation, 23 (2014a), 279-299.

Stephens, Eric and James R. Thompson, "Separation Without Exclusion in Financial Insurance," Journal of Risk and Insurance, Forthcoming (2014b).

Thompson, James R., "Counterparty Risk in Financial Contracts: Should the Insured Worry About the Insurer?," Quarterly Journal of Economics, 90 (2010), 1195-1252. 